\title{
Late deceleration and hypoxia index in fetal monitoring
}

\author{
Kazuo Maeda* \\ Department of Obstetrics and Gynecology, Emeritus, Tottori university, Medical School, Yonago, Japan
}

\begin{abstract}
Late deceleration (LD) of fetal heart rate (FHR) was analyzed to clarify controversy LD record. The mechanical compression of pelvic large arteries by contracted uterus develops LD, and the pathologic fetal hypoxia is enhanced to developed pathologic outcome in frequently repeated LDs. 3 typical pattern LD resulted fully normal outcome of which Apgar was 9, while repeated LD for 50 minutes developed severe asphyxia of which Apgar was 3 and accompanied severe fetal brain damage, in addition, $\mathrm{LD} \backslash$ is defined after 15 minutes repetition. It is concluded from the facts that $\mathrm{LD}$ is ominous due to its frequent repetition but not by its late appearance pattern. Base on the facts, fetal damage is estimated by the hypoxia index (HI), which is the sum of durations (min) of FHR deceleration (transient bradycardia) divided by the lowest FHR (bpm) and multiplied by 100 to keep the integer. The hypoxia index was 25 or more in intrapartum fetal monitoring of all 6 cerebral palsy cases, while it was 24 or less in all 16 cases of no cerebral palsy. The probability to be error is zero, if it is said that cerebral palsy is prevented when the $\mathrm{HI}$ is 24 or less.
\end{abstract}

\section{Introduction}

FHR deceleration (D) was classified to periodic D, including early and late Ds, and variable Dmild \& severe variable D including mild and severe variable Ds, in FHR pattern classification, where LD and severe VD are ominous [1,2], but the author experienced controversy facts, where 3 connected typical LDs resulted fully normal outcome with Apgar 9, while frequently repeated LDs in 50 min (Figure 1) resulted severe asphyxia and heavy brain damage causing infantile brain hemorrhage \& death. In addition, there was a LD definition, where LD was diagnosed after 15 minutes repartition. Thus, it was concluded that LD is not ominous due to its particular pattern which was later appearance than contraction, but its frequently repeated appearance is ominous, because FHR bradycardia appears in fetal hypoxia, where the vagal nerve center of medulla oblongata is stimulated by hypoxia, that was confirmed by the linear relation of heart rate to arterial $\mathrm{PaO} 2$ lower than 50nnHg [2] and human fetal $\mathrm{PaO}_{2}$ is 50 or less $\mathrm{mmHg}$ [3].

The author intended to study the mechanism to develop LD in past reports [2-4]. The developing mechanism of LDs was the compression of maternal pelvic iliac vessels with contracted uterus prior to the FHR deceleration with lag time, while the LD disappeared by the change of maternal posture to lateral one removing the pressure of contracted uterus [3].

\section{Methods}

The situations to develop delayed FHR deceleration after uterine contraction in $\mathrm{LD}$ was reported in past literatures, where the contracted uterus in the labor compressed maternal pelvic iliac artery to stop placental maternal circulation causing fetal hypoxia was shown in pelvic angiography in the labor, and late deceleration disappeared by changing maternal posture to lateral one from supine [1-4], where the fact was that LD damages fetal outcome by its frequent repetition, but not by its particular late appearance after contraction, so that, novel hypoxia index is the sum of deceleration duration $\mathrm{s}$ ( $\mathrm{min}$ ), divided by the lowest FHR (Figure 2) and multiplied by 100 to keep the integer.

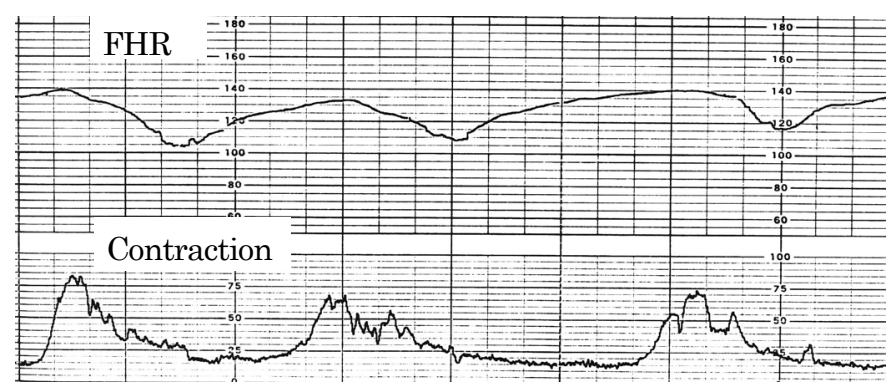

Figure 1. Typical LD and the loss of variability in the labor. Fetal hypoxia index was 26 One min Apgar score was 3. The infant died due to brain hemorrhage

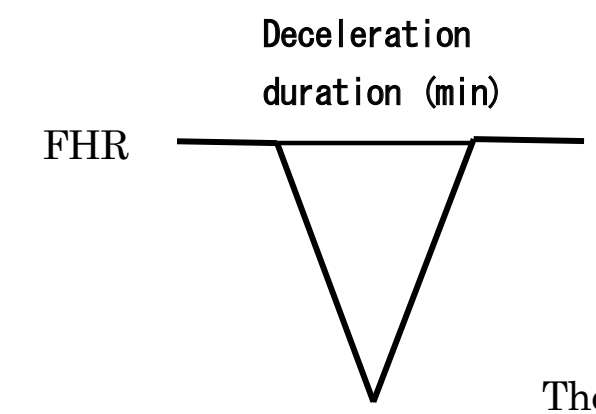

The lowest FHR

Figure 2. The determination of deceleration duration (min) and the lowest FHR (bpm) in hypoxia index

${ }^{\star}$ Correspondence to: Kazuo Maeda, Department of Obstetrics and Gynecology, Emeritus, Tottori university, Medical School, Postal: 3-125, Nadamachi, Yonago, Tottoriken, 683-0835 Japan, Phone and FAX: 81-859-22-6856; E-mail: maedak@mocha.ocn.ne.jp

Key words: fetal heart rate, late deceleration, hypoxia index, cerebral palsy

Received: November 26, 2018; Accepted: December 07, 2018; Published: December 14, 2018 
As the hypoxia index incorporates no lag time in the calculating equation, it is applied to all kinds of decelerations, namely, the application to allofearly, late and variable decelerations and continuous bradycardia,

\section{Results}

The method to evaluate FHR changes was hypoxia index, and the target disease was infantile cerebral palsy of which no reduction was blamed in Dublin trial to estimate fetal monitoring effect. This report is the first trial to detect fetal damage with numeral threshold. Present problem was the reduction of cerebral palsy, which has been eagerly wanted to numerically detect with fetal monitoring. We collected 6 cases of cerebral palsy and 16 cases of no cerebral palsy as the control group. The infants were diagnosed in pediatric clinics, where 6 cases were cerebral palsy and 16 cases were no cerebral palsy.

The FHR records were filed in obstetric ward which were analyzed with novel hypoxia index by corresponding author in blind condition.

The hypoxia index was 25 or more in all of 6 cases of cerebral palsy, and 24 or less in all of 16 no cerebral palsy cases (Table 1 ). The number of cerebral palsies was analyze with the $\chi^{2}$ test calculating the probability to incorrectly diagnose no cerebral palsy to cerebral palsy. The $\chi^{2} \mathrm{p}=$ $0.000008<0.05$, significant difference, where the probability to make error was almost zero, so that we say that cerebral palsy is prevented, if the hypoxia index is 24 or less (Table 1).

\section{Discussion}

\section{Compression of blood vessels}

1. Supine hypotension: Inferior vena cava was compressed by contracted pregnant uterus reducing the blood return to maternal heart and developed maternal hypotension followed by the reduction of placental arterial blood flow, developing fetal hypoxia and FHR deceleration. It is well known that the supine hypotension disappears after changing maternal posture to lateral one from the supine.

2. Late deceleration: Pelvic large iliac arteries were compressed by contracted uterus in the labor at supine posture, stopping placental arterial blood supply, developing fetal hypoxia and the deceleration after contraction (Poseiro effect). The pelvic angiography revealed the fact. The LD disappeared after maternal lateral posture [3].

3. Umbilical cord vessel compression: It develops fetal hypoxia and deceleration. The deceleration disappears if the circulation recovers to normal. Thus, maternal posture change is tried also in suspected umbilical cord compression. The facts show the usefulness of maternal posture change in fetal hypoxia [5].

\section{The loss of FHR accelerations in early stage of fetal hypoxia}

Since fetal brain reacts fetal movement developing FHR acceleration (transient tachycardia.), that is the same as adult physiology. As fetal brain function is suppressed in the hypoxia, FHR acceleration is lost against fetal movement burst (grouped movement signals). The loss of acceleration seems reversible and the baseline variability is preserved in the loss of acceleration, while it should be careful that the most heavy fetal brain damage, which is the loss of FHR baseline variability followed by cerebral palsy, develops within 2 weeks after the loss of acceleration, thus, it may be reasonable to perform early caesarean delivery, when the loss of acceleration appears.

\section{The loss of FHR variability}

Fetal outcome was ominous even in very mild LD by Hon [1], however, mild LD was recognized when FHR variability is lost [1]. As the loss of variability was the mostly severe fetal brain damage similar to anencephaly [6], as fetal brain dose not respond to minor FHR changes. Thus, fetal asphyxia is treated by early C-delivery before the loss of variability, that means to deliver the fetus when the hypoxia index is 24 or less, not after the loss of variability that develops cerebral palsy.

\section{Causes of fetal hypoxia}

1) Fetal disorders: Congenital or acquired fetal diseases develop fetal hypoxia and FHR deceleration, because the heart rate is parallel to $\mathrm{PaO}_{2}$ when il is lower than $50 \mathrm{mmHg}$, which is common level of fetal arterial blood.

2) Extrinsic causes: Low maternal blood pressure, maternal hypoxia, abnormal placental oxygen transfer function, e.g. infarction in preeclampsia, intervillous space fibrin deposit, umbilical cord compression or uterine hypercontraction.

3) Compression of maternal and fetal vessels

\section{Maternal supine hypotension}

Maternal inferior vena cava is compressed by contracted pregnant uterus in maternal supine posture causing maternal hypotension by reduced blood return to the heart and fetal hypoxia causing FHR deceleration, which disappears after change of maternal posture to lateral one.

\section{Compression of iliac arteries}

Contracted pregnant uterus in maternal supine posture stops material blood supply to the placenta causing fetal hypoxia. The mechanism was confirmed by pelvic angiography (Poseiro effect). Fetal deceleration occurs after the lag time, namely, late deceleration (LD) appears, where the LD disappears after maternal posture change to lateral one [3].

\section{Umbilical vessel compression}

It appears in the cord prolapse, lower presenting cord, nuchal cord, compression by fetal body and so on. Maternal lateral posture may effective in conservative therapy. Heavy case receives caesarean delivery.

\section{Management of FHR decelerations}

As maternal lateral posture rejects the pelvic vessel compression caused by contracted pregnant uterus in maternal supine posture, i.e., the vessel compression is removed by maternal lateral posture in the case of FHR deceleration increasing the blood supply to the placenta. As the effect of lateral posture is revealed by quick disappearance of deceleration [4], the mother has to take lateral posture immediately after recording deceleration in fetal monitoring not to increase hypoxia index. Other cause should be checked, if the deceleration is still present after the lateral posture.

\section{Tocolysis will be done in the uterine hypercontraction}

Orcinoprenaline was effective to the LD in uterine hyper contraction in the old report [4]. The tocolysis will be effective by the terbutaliine at present. 


\section{Early delivery}

Severe LD case is cured by early delivery with C-section before the loss of variability preventing fetal brain damage, when intrauterine therapy was ineffective. Timely C-section will be performed before the loss of FHR variability, which is the sign of heavy fetal brain damage resulting neurological sequels, e.g. cerebral palsy [6]. Timely C-section will be indicated by severe FHR changes, the loss of FHR acceleration, decreased variability [6], or when the hypoxia index was 20-24, as the index of cases of the loss of variability was 25-26. The index is determined by the sum of FHR bradycardia duration (min) x100, divided by the lowest bradycardia (bpm), because FHR lower than 110 bpm will be parallel to $\mathrm{PaO}_{2}$ lower than $50 \mathrm{mmHg}$.

\section{Actocardiogram}

The neonate was vigorous when fetal acceleration duration ratio to fetal movement burst duration (A/B ratio) was higher than 1.0, despite repeated LDs were recorded, namely, fetal movement study was effective to estimate favorable outcome even in the presence of LD [7].

\section{Possibility of fetal/neonatal damage}

Although FHR is parallel to the $\mathrm{PaO} 2$ under $50 \mathrm{mmHg}$, where fetal bradycardia is recorded in monitoring record, it is not the same as fetal damage, namely, the bradycardia is caused by the neurologic excitation of parasympathicus center located in the medulla oblongata, and the experimental hypoxic bradycardia disappears after the narcosis of rabbit, and the apneic bradycardia of anencephalic newborn who remains medulla oblongata disappeared by the infusion of oxygenated blood into the vessel. Fetal brain is damaged when FHR acceleration is lost an the variability lost by the hypoxia, namely fetal bradycardia shows only fetal environmental hypoxia, while hypoxic fetal brain damage is expressed by the loss of fetal brain response to fetal movement, i.e. FHR acceleration and the variability are fetal brain response to fetal movement burst and minor fetal movements, therefore the loss of acceleration to fetal movement is early sign of fetal brain damage, while the loss of variability is advanced severe fetal brain damage, confirmed by actocardiographic studies [6,7]. Therefore, the early appearance of LD caused by the compression of large vessels which recovered after posture change cannot be fetal damage, therefore, the neonate is vigorous without brain damage, while sum of hypoxic effects in long repetition may be able to damage fetal brain if the LD lasted for several $10 \mathrm{~min}$. The fetal damage could be estimated by the hypoxia index, which is the sum of duration of fetal bradycardia (min), multiplied by 100, and divided by the lowest FHR (bpm). As the Index after the loss of viability was $25-26$, it must be around 20 , if fetal brain damage would be prevented. However, that is only an example of extreme case, and the author hope rapid cure of LD case in its earliest stage, e.g. the cure from LD caused by uterine body compression. Actocardiographic duration to fetal movement duration

\section{Conclusion}

There would be several kinds in LD. Maternal posture change to lateral one should be tried after looking at LD on monitoring chart. Although final decision will be cesarean section, as early as possible intrauterine therapy would be tried. The actocardiogram will be helpful to expect fetal outcome by the FHR acceleration duration ratio to the duration of fetal movement (A/B ratio), i.e. the outcome is favorable, if the $\mathrm{A} / \mathrm{B}$ ratio is larger than 1.

\section{References}

1. Hon EH. An atlas of fetal heart rate patterns. Harty Press, New Haven, 1968.

2. Caldeyro-Barcia R, Poseiro JJ, Mendez-Bauer C, Gulin LO (1967) Effects of Abnormal Uterine Contractions on Fetal Heart Rate in Labor. 1967, 9-27.

3. Poseiro JJ, Mendez-Bauer C, Caldeyro-Barcia R, Pose SV (1969) Effect of uterine contractions on maternal blood flow through the placenta. Perinatal factors affecting human development, Paho Advisary Committee. 161-171.

4. Caldeyro-Barcia R, Magana JM, Poseiro, JJ, Castillo JB, Pose SV, et al. (1969) New Approach to the treatment of acute Intrapartum fetal distress. Perinatall factors affecting human development. Patho Advisary Committee. 248-253.

5. Teshima N (1993) Non-reactive pattern diagnosed by ultrasonic Doppler feta actocardiogram and outcome of the fetuses with non-reactive pattern. Acta Obstet Gynecol Jpn 45: 423-430.

6. Maeda K (2014) Modalities of fetal evaluation to detect fetal compromise prior to the development of significant neurological damage. J Obstet Gynaecol Res 40: 20892094. [Crossref]

7. Maeda K (2016) Fetal Actocardiogram?Analysis of Fetal Motion and Heart Rate, Jaypee Brothers, New Delhi, 2016.

Copyright: (C2018 Maeda K. This is an open-access article distributed under the terms of the Creative Commons Attribution License, which permits unrestricted use, distribution, and reproduction in any medium, provided the original author and source are credited. 